\section{RÉPLICA: MENSAJES DE TEXTO PARA MEJORAR LA ADHERENCIA AL TRATAMIENTO DE PERSONAS QUE VIVEN CON EL VIH}

\section{REPLY: TEXT MESSAGES TO IMPROVE TREAT- MENT ADHERENCE IN PEOPLE LIVING WITH HIV}

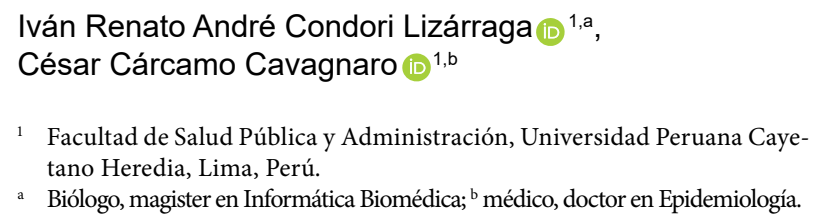

1 Facultad de Salud Pública y Administración, Universidad Peruana Cayetano Heredia, Lima, Perú.

a Biólogo, magister en Informática Biomédica; ${ }^{\text {b }}$ médico, doctor en Epidemiología.

Sr. Editor: Agradecemos el comentario y la ampliación de la revisión bibliográfica, sobre todo del artículo de Pop-Eleches et al. ${ }^{(1)}$.

En las primeras revisiones de la bibliografía, se encontró el artículo de Da Costa et al. (2), el cual fue un estudio con mujeres de Brasil, dónde la muestra fue de 21 y la intervención fue solo con ocho mujeres. Consideramos que la muestra fue muy pequeña para incluirlo como referencia.

Se mencionó también unos datos de Guatemala ${ }^{(3)}$, sin embargo, al realizar la búsqueda en diversas fuentes no se encontró el acceso a dicha información.
Si bien existen múltiples evaluaciones relacionadas a nivel mundial, la presentada es la primera en el Perú que evalúa un resultado objetivo, debido a que la tesis del Dr. Curioso ${ }^{(4)}$ presenta la medición de la adherencia de forma autorreportada. Esperamos esa tesis sea publicada pronto en una revista científica.

\section{REFERENCIAS BIBLIOGRÁFICAS}

1. Pop-Eleches C, Thirumurthy H, Habyarimana JP, Zivin JG, Goldstein MP, de Walque $\mathrm{D}$, et al. Mobile phone technologies improve adherence to antiretroviral treatment in a resource-limited setting: a randomized controlled trial of text message reminders. AIDS 2011;25(6):825-34.

2. Da Costa TM, Barbosa BJ, E Costa DA, Sigulem D, de Fatima Marin $\mathrm{H}$, Filho AC, et al. Results of a randomized controlled trial to assess the effects of a mobile SMS-based intervention on treatment adherence in HIV/AIDS-infected Brazilian women and impressions and satisfaction with respect to incoming messages. Int J Med Inform. 2012;81(4):257-69.

3. Ikeda JM, Barrios R, Lopez-Lopez JB, Hearst N. SMS messaging improves treatment outcome among the HIV-positive Mayan population in rural Guatemala. In: XIX International AIDS Conference, Washington, 22-27 July 2012, \#TUPE673 (Poster).

4. Curioso WH. Evaluation of a Computer-Based System using Cell Phones for HIV positive people in Peru [Tesis Doctoral]. Washington: Biomedical Informatics and Medical Education, University of Washington; 2012. Disponible en: https://digital.lib.washington.edu/ researchworks/handle/1773/20614

\footnotetext{
Citar como: Condori Lizárraga IRA, Cárcamo Cavagnaro C. Replica: mensajes de texto para mejorar la adherencia al tratamiento de personas viviendo con el VIH. Rev Peru Med Exp Salud Publica. 2020;37(1):180. Doi: https:// doi.org/10.17843/rpmesp.2020.371.5221
} Correspondencia: Iván Condori; Av. Bolognesi 676 Sta Anita, Lima, Perú;
ivan.condori.l@upch.pe 